\title{
APURDUTM
}

Euskal ikerketen aldizkaria | Revue d'études basques |

Revista de estudios vascos | Basque studies review

$3 \mid 1998$

Numéro III

\section{R. L. Trask. The history of Basque.}

Routledge : Londres et New York, 1997, XXII + 458 p. ISBN : 0-415-131

16-2 (hbk). Environ $650 \mathrm{FF}$.

\section{Bernard Oyharçabal}

\section{OpenEdition}

\section{Journals}

Édition électronique

URL : https://journals.openedition.org/lapurdum/1748

DOI : 10.4000/lapurdum. 1748

ISSN : 1965-0655

Éditeur

IKER

Édition imprimée

Date de publication : 1 octobre 1998

Pagination : $317-333$

ISBN : 2-84127-152-8

ISSN : $1273-3830$

Référence électronique

Bernard Oyharçabal, «R. L. Trask. The history of Basque. », Lapurdum [En ligne], 3 | 1998, mis en ligne le 01 septembre 2010, consulté le 24 février 2022. URL : http://journals.openedition.org/lapurdum/1748 ; DOI : https://doi.org/10.4000/lapurdum. 1748 


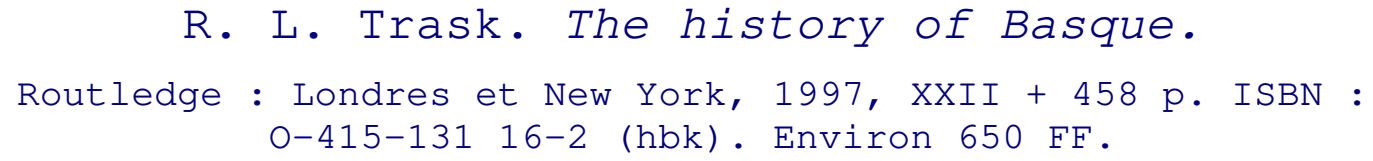

L. R. Trask est un linguiste américain, enseignant au Royaume-Uni (University of Sussex), dont les études concernant l'euskara sont justement reconnues parmi les bascologues. Ses recherches l'ont également conduit à réaliser des travaux de linguistique générale, parmi lesquels nous citerons en particulier, à côté d'une introduction à la variation diachronique dans les langues (Linguistic Change, 1994) et d'un excellent manuel de linguistique historique (Historical Linguistics, 1996), deux dictionnaires de linguistique fort utiles (A dictionary of Grammatical Terms in Linguistics, 1993, et A Dictionary of Phonetics and Phonology, 1996).

L'ouvrage dont nous rendons compte ici, intitulé L'histoire de la langue basque, a pour objectif premier d'offrir aux comparatistes et linguistes non spécialistes du basque l'information indispensable concernant son histoire interne, laquelle, observe l'auteur, se trouve le plus souvent tout simplement ignorée, ou inexplicablement négligée, en dehors des bascologues, alors même que la langue basque depuis deux siècles a donné l'occasion à de multiples reprises à des rapprochements et comparaisons génétiques. Mais il va sans dire que les spécialistes liront également avec profit ce travail, très riche en informations et suggestions diverses, souvent stimulantes. A cet égard, on ne peut que se féliciter de la parution de cet ouvrage de 458 pages (+XXII p.), bien informé sur les questions linguistiques dont il traite à titre principal, clair, et solidement structuré autour de six chapitres traitant dans le détail de l'histoire de la langue dans ses divers aspects, phonologie (ch. 3), grammaire (ch. 4), lexique (ch. 5), relations avec d'autres langues (ch. 6), précédés d'une longue introduction (ch. 1), et d'une vue générale des caractéristiques de la langue (ch. 2). Se situant clairement dans la lignée des travaux de Michelena, même si l'auteur n'hésite pas à formuler de temps à autre ses propres hypothèses, cette histoire de la langue basque est appelée à devenir un ouvrage de référence dans le domaine des études diachroniques basques et des recherches de comparaison génétique. Pour cette raison, le lecteur excusera un compte rendu critique plus détaillé qu'il est de coutume.

L'introduction destinée à présenter le Pays Basque aux lecteurs peu familiers de son histoire est relativement longue (81 p.). S'il faut féliciter l'auteur de son effort en vue de présenter aux lecteurs non familiers du Pays Basque la langue dans son environnement, ce chapitre est aussi, à notre avis, le moins réussi de l'ouvrage. Après quelques brèves considérations sur la situation géographique, et sociologique de la langue et de ses dialectes, il comporte un résumé de l'histoire du Pays Basque (p. 7-35), dans lequel on regrettera la présence d'approximations qui auraient pu être évitées, même si l'auteur n'est pas un spécialiste d'histoire. Par exemple, la fin du Royaume de Navarre, conquis par la couronne de Castille au début du $16^{\mathrm{e}}$, est présentée comme une absorption tranquille, pratiquement passée inaperçue (p. 19), alors qu'elle fut l'occasion d'un long et pénible conflit, très cruel pour les populations, et qui exacerba tout au long du $16^{\mathrm{e}}$ siècle et encore au siècle suivant, jusqu'au Traité des Pyrénées, la rivalité franco-espagnole. 
Au surplus, il aboutit à la séparation de la Basse-Navarre du reste de la Navarre, événement qui n'est pas rapporté dans cette introduction, malgré le poids relatif de ces circonstances qui entourent la naissance de la littérature basque, puisque les premiers écrivains basques eurent à en connaître directement ou développèrent leur oeuvre basque dans ce contexte : Dechepare, qui fut emprisonné lors de ce conflit, sans doute pour ses sympathies pour la couronne de Castille ; Liçarrague qui engagea sa traduction du Nouveau Testament à la requête de Jeanne d'Albret, Reine de Navarre, laquelle, empêchée de régner sur la plus grande partie du Royaume, ne s'était pas résignée à cette amputation. La période révolutionnaire est aussi évoquée de manière caricaturale, avec au surplus une étonnante référence à de nouvelles lois interdisant l'usage des langues régionales (p. 21), qui n'ont pas existé. Pour la période plus récente, l'auteur offre un rappel, inattendu et, à mon sens, hors de propos, des événements politiques survenus depuis un demi-siècle (lutte de l'ETA, actions du GAL, etc.), et incluant parfois des informations erronées, comme la mention (p. 32) d'un référendum qui aurait eu lieu en Navarre concernant l'intégration à la Communauté autonome basque. La situation politico-administrative et institutionnelle actuelle du Pays Basque, et, surtout, le dispositif juridique existant dans ses différentes parties en ce qui concerne le statut de la langue basque, sont, par contraste, très sobrement indiqués (p. 1). Il s'agit pourtant là, on le sait désormais, d'un point important pour l'avenir immédiat, et à plus long terme, de la langue, et l'expérience en cours en Pays Basque péninsulaire depuis une vingtaine d'années méritait certainement dans cette introduction plus d'attention. De même on regrettera le manque d'informations sur la situation sociolinguistique actuelle, assez bien connue désormais (Aizpurua 1995, Oyharçabal 1997). Plus grave, c'est dans cette introduction que les informations générales relatives aux dialectes figurent à titre principal (p. 5 et 7), mais, déplorons-le, de façon nettement insuffisante dans un tel ouvrage.

La section suivante, consacrée à l'histoire externe de la langue (pp. 35-49), est plus intéressante, même si elle souffre de quelques carences pour la période moderne $\left(16-20^{\mathrm{e}}\right.$ siècle). Pour l'époque de la romanisation, Trask, à l'encontre de ce qui a été longtemps admis, mais conformément à un point de vue largement répandu aujourd'hui parmi les spécialistes, met en doute l'idée que les Caristii, les Autrigons et les Berones et même les Varduli, qui occupaient la plus grande partie des territoires de la Biscaye, du Guipuscoa et de l'Alava actuels, aient été des populations bascophones (p. 36-38). Les éléments de preuve ne sont pas décisifs, comme l'auteur l'admet lui-même, puisqu'ils sont fondés essentiellement sur les témoignages scripturaux recueillis, et où la présence basque est extrêmement réduite. Trask n'ignore pas que les patronymes recueillis de cette façon témoignent surtout de la langue socialement dominante, mais la toponymie étant également plus indoeuropéenne que basque, il opte donc plutôt en faveur de la thèse d'un basquisation tardive de l'ouest du Pays Basque actuel (cf. aussi pour une présentation plus réservée de cette thèse, Gorrochategui 1995) ; Trask (p. 38) n'exclut pas cependant tout à fait l'idée de territoires linguistiquement hétérogènes avec une certaine présence du basque au temps de la romanisation, comme cela est admis pour la Navarre. Sont ensuite rapidement examinés les principaux témoignages écrits de la langue, de façon très elliptique à partir du $16^{*}$ siècle, et sans que l'auteur prenne la peine d'exposer, avec la précision souhaitable, ce que l'on sait, et qui n'est pas négligeable, du recul territorial de la langue durant ces derniers siècles. Néanmoins cette carence est compensée par la présence d'une carte montrant le recul des frontières de la langue dans sa bordure méridionale, entre le $10^{\mathrm{e}}$ et le $19^{\mathrm{e}}$ siècle (p. 4). 
On ne voit pas, par contre, ce qui a présidé aux choix apparaissant dans un parcours plus que cursif sur une partie de la littérature basque, d'autant que, là aussi, on doit constater des approximations ou des anachronismes, certes de peu de conséquences, mais malgré tout gênants pour un ouvrage de référence destiné éventuellement à un public qui n'aura pas toujours aisément accès aux sources de première main : $\mathrm{B}$. Dechepare est déclaré Basque français (p. 47), et le livre de navigation de Hoyarzabal (mentionné d'après une réédition basque de 1677, et cité ici, alors que les écrits en basque d'Etcheberri de Sare ne sont pas mentionnés) est attribué (p. 48) à Martin de Hoyarzabal et Piarres Detcheverry alors que le premier est l'auteur (en français ; $1^{\text {re }}$ édition 1577) de l'ouvrage, et le second son traducteur (bien que cette traduction soit légèrement augmentée ; $1^{\text {re }}$ édition 1633). La présentation générale consacrée aux études bascologiques évite heureusement de tels écueils. Les principales contributions à ces études sont mentionnées, et pour la période moderne l'apport considérable de Michelena bien souligné, ainsi que ceux d'Azkue et Lafitte. Dans son évocation du dictionnaire de S. Pouvreau, l'auteur analyse le vocable ibai dans le syntagme ur ibaia, 'gué, rivière guéable', comme un adjectif modifiant ur (p. 51). Puisque la traduction est ambiguë [gué/guéable], et qu'ibai n'est attesté par ailleurs que comme substantif, il paraît malgré tout plus simple d'y voir un composé $[\mathrm{N}+\mathrm{N}]$ où ibai serait, non un adjectif, mais un nom signifiant 'gué', comme cela est rapporté par Moret en Navarre ainsi que l'indique Michelena (1961), cf. aussi OEH-DGV IX (p. 103). Au delà de ce détail, on saura gré à l'auteur d'avoir souligné la notable influence, souvent ignorée (mais que je crains quelque peu exagérée ici, malgré tout), des idées de J. D. Garat sur Humboldt (p. 53). De façon générale, peu d'oublis doivent être constatés ; on regrettera néanmoins l'absence de mention pour les grammaires suivantes: Harriet (ouvrage publié en 1741, se voulant une grammaire du français, mais première grammaire publiée écrite en basque et qui servit de base documentaire à divers travaux postérieurs), Lécluse (1 826), Darrigol (1 827), d'Abbadie \& Chaho (1836). La contribution de Martinet au débat sur l'analyse de l'ergativité en basque (p. 66) est sévèrement jugée (alors que ses mérites dans le domaine de la phonologie historique sont eux reconnus). Certes les propositions de Martinet concernant l'ergativité en basque (Martinet 1958) ressemblaient un peu à un refus de syntaxe et il est exact qu'elles n'eurent guère d'écho hors de France, malgré N'Diaye (1970), Tchekhoff (1978) et tout récemment, dans le même cadre fonctionnaliste, Coyos (1998). Il faut lui savoir gré, toutefois, d'avoir mis en question dès les années 50, cette théorie de la passivité qui apparaissait après-guerre comme un dogme intangible parmi les linguistes et bascologues non natifs, et qui ne sera pas abandonnée pratiquement avant les années 1970.

Le second chapitre est consacré à une présentation des caractéristiques de la langue. Le système phonologique est bien présenté avec des indications générales regardant les variétés dialectales. On s'étonnera malgré tout de l'indication selon laquelle l'opposition /r/ vs / rr/ à l'intervocalique tend à se perdre en labourdin et bas-navarrais, avec une réalisation de plus en plus fréquente, quoique non systématique, nous dit l'auteur, de $/ r /[R]$ (p. 85). A ma connaissance, cette observation, que Trask répétera au chapitre 3 (cf. infra), n'est pas exacte, l'opposition se maintenant toujours dans cette position sans exception non explicable chez les locuteurs natifs. La perte de l'opposition entre les phonèmes retranscrits dans l'orthographe ordinaire s vs x, et de celle de leurs correspondants affriqués, observable dans les jeunes générations dans ces dialectes, à l'inverse, n'est pas mentionnée ; il est vrai qu'elle n'a pas été jusqu'ici l'objet de publications. La structure du syntagme nominal est clairement exposée, quoiqu'il faille ici aussi regretter un jugement peu charitable, et pour tout dire excessif, concernant les travaux existants: Presque sans exception, les grammaires du basque disponibles offrent une image désespérément confuse des syntagmes nominaux, dont l'existence le plus souvent n'est même pas reconnue (p. 89). 
Apparemment les références de l'auteur ont quelque peu vieilli. Il est exact que la grammaire de Lafitte (1944, $2^{\mathrm{e}}$ édition corrigée en 1962), longtemps principale référence pour les linguistes non natifs, ignore cette notion, pourtant très importante en basque, car l'article et les cas se joignent, une seule fois, au dernier élément sur la droite du groupe nominal. Toutefois depuis un quart de siècle, pour le moins, pratiquement tous les travaux de grammaire précisent clairement les choses. Elles sont d'ailleurs déjà exposées dans un petit ouvrage se voulant une version simplifiée de la grammaire de Lafitte : la grammaire basque d'Arotçarena (1948) où la désignation employée est celle de bloc fonction.

Les éléments du syntagme nominal sont bien présentés, même si l'on regrettera une analyse des quantifieurs comme des déterminants, ce qui conduit à l'idée peu satisfaisante, et mal commode, selon laquelle tout SN peut avoir deux déterminants occupant des positions très différentes à droite et à gauche du nom-tête (p. 89). Une telle vue rend pratiquement inopérant pour le basque la notion de syntagme du déterminant (SD) aujourd'hui souvent admise en tant que projection fonctionnelle associée aux syntagmes nominaux. La reconnaissance d'un seul déterminant, occupant la dernière position à droite du SN, et correspondant soit à l'article défini, soit à un démonstratif ou un déterminant indéfini (bat 'un', ou $\varnothing$, et, en suivant volontiers Trask sur ce point, le partitif), permet au contraire d'accorder sa pleine place à la notion de SD, et d'ouvrir la voie à une meilleure approche typologique (tête fonctionnelle à droite des projections lexicales compléments) et à une meilleure description des données empiriques (Artiagoitia 1997).

Dans l'exposition du système casuel, Trask retient une douzaine de cas. Il exclut notamment le partitif (qu'il préfère considérer, nous venons de le signaler, comme un déterminant indéfini), ainsi que le suffixe d'adnominalisation $-k o$, regrettant à juste titre l'emploi inadéquat dans la tradition grammaticale de la notion de génitif locatif (p. 102), proposition qu'il avait soutenue dans un article antérieur (Trask 1985b). Certaines inexactitudes doivent être relevées: il est dit qu'un ou deux verbes régissent le cas instrumental (p. 92), alors que cela est vrai, par exemple, de la plupart des verbes de communication, pensée, opinion, utilisation, jouissance, etc... autrement dit un nombre relativement important de verbes (cf. infra). A vrai dire, Trask part du principe que le cas instrumental exprime premièrement les instruments (p. 92). Ceci est très douteux, car s'il est une situation où, en basque contemporain, la nature casuelle du suffixe dit instrumental $-\mathrm{z}$ est peu claire, c'est lorsqu'il a une valeur strictement instrumentale (au sens où il marque l'instrument par lequel le procès que décrit le verbe est accompli). En effet, sauf exceptions bien limitées (impliquant certains organes corporels), le suffixe n'apparaît alors d'ordinaire qu'à l'indéfini, et le plus souvent joint à des noms simples (dailuz 'à la faux', aizkoraz 'à la hache', grekoz 'en grec', ...). Si le syntagme est doté d'un déterminant défini et de modifieurs du nom-tête, l'emploi du suffixe instrumental devient non naturel : La question *Zertaz moztu duzu egurra ? 'Avec quoi avez-vous coupé le bois'ou la phrase *Coizean erosi aizkora berri horretaz moztu dut egurra 'J'ai coupé le bois avec cette hache achetée ce matin' sont, ou mal formées ou très douteuses, mais n'offrent pas de difficultés si l'instrument est décliné au comitatif (zerekin 'avec quoi', aizkora berri horrekin dans les exemples). Il est simplement erroné de voir dans cet emploi du comitatif un usage substandard des jeunes locuteurs (p. 92). 
Au demeurant, puisque l'auteur semble considérer, judicieusement à notre avis, que seuls les suffixes pouvant se joindre à des SD doivent être considérés comme des cas (p. 98), sans doute vaudrait-il mieux dire que, dans cet usage instrumental, -z correspond à un suffixe de dérivation adverbial (comme $-k a$, par exemple), et qu'il ne représente un véritable cas que dans les emplois où il peut être librement joint à des SD, par exemple lorsqu'il est régi par un verbe de communication (mintzatu, hitz egin 'parler', erran 'dire',...), réflexion ou imagination (gogoeta egin 'réfléchir', pentsatu 'penser', amets egin 'rêver', ...), opinion (uste izan 'croire'), et quelques autres comme baliatu 'utiliser, tirer profit', aberastu 's'enrichir', jabetu 's'approprier', gabetu '(se) dépouiller', etc.

La morphologie verbale n'appelle pas de commentaires particuliers. Observons pourtant la présence de quelques erreurs comme dans l'exemple construit bizi- $\emptyset$ ahal dute 'ils peuvent vivre' (p. 109), donné en variante dialectale du régulier bizitzen ahal dira, mais qui est mal formé.

Le problème de la valence est exposé de façon claire, mais, à notre gré, passablement vieillie. Les formes à indice d'ergatif sont ainsi considérées comme des transitives, même si l'auteur reconnaît l'existence de diverses constructions où ces formes ne peuvent avoir d'objet direct et sont donc syntaxiquement intransitives (p. 111). Mais aucune référence explicite n'est faite à la distinction entre verbes inaccusatifs et inergatifs, souvent admise aujourd'hui, et introduite dans les études basques depuis Levin (1983). Il est vrai que l'auteur rejette dans le chapitre 4 les éléments d'analyse (surtout ceux de nature sémantique) liés à la distinction entre les deux types d'intransitifs (p. 230), point de vue qui explique également pourquoi les verbes composés à objet incorporé du type [ $N$ egin ' $\mathrm{N}$ faire'], typiquement inergatifs, sont à peine cités à côté de quelques verbes 'déponents' (au sens de Lafitte). Par ailleurs, Trask refuse de considérer comme des constructions formellement passives celles où la forme conjuguée du verbe s'accorde avec l'objet direct, l'agent demeurant absent soit de façon obligatoire (médiopassives), soit optionnelle (passives du parfait résultatif) (p. 112-113) ; (cf. dans la même optique, Ortiz de Urbina et Uribe-Etxeberria, 1991)). Néanmoins l'argumentation présentée par Trask n'emporte pas la conviction : dire que le complément d'agent est argument du seul verbe participe ne démontre rien, car il est bien connu que si certaines langues ont un passif synthétique, comme, par exemple, le latin, d'autres ne connaissent qu'un passif périphrastique présentant le verbe être associé à un participe perfectif, dont l'argument agentif correspond au complément d'agent. D'autant que la littérature atteste abondamment de l'existence de telles formes avec un complément d'agent réalisé sous la forme d'un syntagme instrumental comme dans l'exemple labourdin suivant : Nahi zare maitatua izan Jainkoaz eta gizonez? 'Voulez-vous être aimé par Dieu et par les hommes ?' voire, dans les dialectes occidentaux modernes, au motivatif suivant le modèle espagnol (Rebuschi 1984: 435). En fait, on ne voit pas très bien à quoi renvoie la distinction entre phrases fonctionnellement passives et phrases formellement passives à laquelle se réfère Trask (p. 113), car aucune définition formelle de la passivation n'est donnée qui permettrait d'exclure la forme basque, attestée dès le $16^{\mathrm{e}}$ siècle. Au surplus les exemples choisis dans cette discussion sont parfois douteux : ainsi l'équivalence entre Lan hori egina da et Lan hori egindakoa da qu'indique Trask (p. 112-113) pour dire Ce travail est fait est très discutable, car dans cette seconde expression le prédicat n'a qu'une interprétation nominale et la phrase a par conséquent une valeur équative (au demeurant progmatiquement difficile) : Ce travail est celui qui a été fait. Cette remarque vaut également pour l'autre exemple de même type offert par Trask (p. 112) : Ibaiak kutsatuak dira / Ibaiak kutsatutakoak dira 'Les rivières sont polluées', où seule la première phrase correspond à la traduction. Les constructions dites antipassives (Rebuschi, 1984) ne sont pas mentionnées. 
Ce deuxième chapitre se termine par un répertoire des particularités typologiques du basque (p. 117-123), listées dans une optique historique à partir de Nichols (1992).

Le chapitre 3 est consacré à la phonologie. Il est d'une grande importance puisque la phonologie constitue l'élément de base dans les études de linguistique historique. Ce chapitre représente un excellent condensé des connaissances actuelles dans ce domaine, lesquelles sont loin d'être négligeables grâce en particulier à la contribution de Michelena. Trask présente le système phonologique reconstruit du basque au moment de la romanisation, et examine l'évolution des différentes séries de phonèmes jusqu'au basque moderne, mettant en évidence (p. 128) les dangers qu'il y a à procéder à des comparaisons de longue distance qui ne tiennent pas compte de ces éléments. Comme l'avait montré Michelena, l'existence d'emprunts directs au latin, puis aux langues romanes, la présence de dialectes offrant des variations régulières, la connaissance d'un corpus onomastique en langue aquitaine, et de documents médiévaux incluant des noms et gloses en basque, permet de procéder à une reconstruction relativement fiable des sons du pré-basque, remontant approximativement à 2000 ans en arrière (p. 125). Le système phonologique de ce basque comprend dans cette présentation (p. 126) les 5 voyelles cardinales (comme en basque moderne pour la plupart des dialectes), et 16 consonnes divisées en deux séries séparées par une opposition fortis vs lenis : $(p), t, k, t z, t s$, $N, L, R$ vs $b, d, g, z, s, n, I, r$. Ceci suit le schéma de Michelena (1961, [1976 : p. 374]), lequel toutefois exclut complètement $\mathrm{p}$ (au sujet duquel Trask précise toutefois que les preuves de son existence en pré-basque sont extrêmement réduites). Trask rappelle sa proposition antérieure (Trask 1985a) d'analyser l'opposition fortis vs lenis du pré-basque en termes de gémination (p. 127), mais il mentionne également les réticences que cette vue a pu faire naître, et en particulier les sévères objections de Hurch (1991). L'aspiration est analysée comme résultant de phénomènes suprasegmentaux. Trask insiste, avec raison, sur l'importance de la reconstruction du système phonologique ancien pour l'analyse comparative externe. Si les éléments rassemblés montrent, comme c'est le cas, que le prébasque ignorait les occlusives orales $(\mathrm{p}, t, k)$ et $\mathrm{m}$ en début de mot (en écartant la proposition de Martinet (1955) en faveur d'un $m b$ ), les comparaisons fondées sur de telles bases devront nécessairement être tenues comme suspectes (p. 128).

Après la présentation du système phonologique dans une perspective diachronique, chaque série de phonèmes est ensuite étudiée, et sa distribution présentée, de façon toujours argumentée, d'abord pour les consonnes (plosives, sifflantes, nasales, latérales, rhotiques, palatales), puis les voyelles, et les glides. L'aspiration, la question des groupements de consonnes et de l'accentuation sont ensuite examinées, et un résumé est offert des principaux changements intervenus lors de l'évolution du pré-basque vers le basque moderne. On notera les arguments plaidant en faveur d'une absence de $m$ (p. 133-135). On ne suivra pas nécessairement l'auteur dans sa proposition d'une opposition $\mathrm{r}$ vs $r r$ réduite à la seule position intervocalique (p. 144). Il semble plutôt que l'opposition ait existé en position finale (comme d'ailleurs encore aujourd'hui en labourdin et bas-navarrais, où un -r simple se maintient comme tel devant pause avec certains vocables comme ur 'eau', barur 'jeûne', ou zur 'bois'), bien qu'elle se soit neutralisée dans la plupart des dialectes'. L'opposition a dû exister également devant n, d'où les nombreux doublets dialectaux : oroitvs orroit 'souvenir', en vs erri 'doigt', are vs arre 'herse', etc.. En effet, dans les parlers où l'aspiration a été perdue alors que l'opposition entre vibrantes simples et fortes s'était maintenue devant $h$, on a obtenu soit un -r-soit un -rr- intervocaliques selon la forme de départ: en 'doigt', oroitu 'se souvenir' et are 'herse' d'un coté, mais une 'or' et urrats 'pas' de l'autre. 
A l'inverse, dans les parlers où la neutralisation de $/ \mathrm{r} /$ et $/ \mathrm{rr} /$ devant $h$ a précédé la perte de l'aspiration, on a orroit 'souvenir', erri 'doigt', arre 'herse' (à côté de urre et urrats qui se maintiennent bien sûr) ; dans ce dernier cas les formes intermédiaires, précédentes la perte de l'aspiration, sont déjà attestées dans les textes labourdins du XVII ${ }^{\mathrm{e}}$ siècle (orrhoit, errhi,...); cf. Michelena (1961/1976: p. 329, n. 5). En souletin, où l'aspiration s'est bien maintenue jusqu'à nos jours et où la vibrante simple est devenue 0 , on a donc : ohit 'souvenir', ehi 'doigt', eho 'moudre, tuer', etc. mais également ürrhentü 'achever', errhauts 'poussière', etc. Ayant signalé l'évolution observée dans la réalisation du $r r$ en labourdin et bas-navarrais (aujourd'hui uvulaire, et non apicale, chez de nombreux locuteurs), Trask revient également sur l'indication déjà mentionnée plus haut, mais inexacte, et résultant apparemment d'une simple impression, d'une perte de l'opposition dans ces dialectes entre la vibrante simple et l'uvulaire, faisant que hari 'fil' et hura 'celui-là' seraient prononcés comme harri 'pierre' et hurra. 'la noisette' (p. 145). Concernant la palatalisation on relèvera l'utile distinction entre palatalisation automatique et palatalisation expressive, avec certaines différences notables bien expliquées. Les évolutions en cours et passées sont relevées. L'examen des voyelles, bien qu'excellent, est un peu elliptique: par exemple, aucune présentation des voyelles nasalisées des dialectes extrême-orientaux n'est proposée, bien que leur présence soit signalée (p. 168). A propos de l'analyse de $\ddot{u}$ souletin, on corrigera la forme üdürü du tableau 3.7. (p. 147), erreur ou coquille pour soul, üdüri 'ressemblance'. A propos de l'assimilation entre voyelles fermées (/, ü, $u)$ du souletin, le cas de bürdüña 'fer'(en place de la forme attendue soul. *burdiña) est justement évoqué (p. 150), à côté de ïtsü 'aveugle'(<*îtsü) et bu(r)ü 'tête' (<*burü). Toutefois, sans autre explication, le lecteur non spécialiste aura du mal à retrouver la double harmonisation qui, comme le proposait Lafon (1933), a dû se produire, la première, avant le passage de $u$ à $\ddot{u}$ en souletin, et conduisant à soul. *burduña $(<$ burdiña); la seconde intervenant après le passage à ü du $u$ prénasal: *burduña> *burdüña>bürdüña. Divers phénomènes morphonologiques sont aussi abordés, notamment ceux liés à la suffixation de l'article -a. La fermeture du e- initial dans les participes en -/' dont la seconde syllabe a pour noyau une voyelle fermée est aussi clairement exposée (p. 154), mais l'un des exemples cités surprend : il s'agit de iduri 'sembler' que l'auteur fait provenir de *eduri. En laissant de côté la question de la métathèse $r$ - $d d$-r , l'analyse est discutable puisque dans les formes personnelles on a dirudi, comme l'on a dirau (avec iraun 'durer') ou digaran (avec igaran 'passer' issu du causatif de igan 'monter'), et non *derudi, comme attendu si la forme de départ était *erudi (à laquelle on préférera *eirudi).

L'analyse du diaphonème orthographié / occupe l'essentiel de la section consacrée aux semi-consonnes. Les divers correspondants phoniques de $j$ ayant été très bien présentés auparavant, avec une carte (p. 86), c'est son apparition comme résultat de e- devant principalement a et $o$, qui est discutée, de façon convaincante, sauf peut être pour la forme *eelgi, que Trask, avec Michelena (1961, [1976 : p. 515]), met à la source de soul, elki et jalgi 'sortir', sans tenir compte des réserves du même Michelena (1961, [1976 : p. 63, n. 7]) sur ce point. L'auteur expose également la difficulté de rendre compte de la non généralisation de la fusion de la fricative palatale /S/ (orthographiée $\mathrm{x}$ ) et de la fricative vélaire / $\mathrm{x} /$ (transcrite / dans l'orthographe ordinaire) en guipuscoan. Dans ce dialecte, /j/ passa à /Z/ (comme en souletin et en haut-navarrais), puis /Z/ passa à /S/ (comme en haut-navarrais), mais ensuite /S/ passa à $/ \mathrm{x} /$ (même phénomène qu'en castillan). Donc en guipuscoan, comme en hautnavarrais, il y eut une étape où le résultat de $/ \mathrm{j} /$ étant $/ \mathrm{S} /$, il y eut fusion avec le phonème $/ \mathrm{S} /$ existant de manière indépendante. 
Mais alors qu'en haut-navarrais on en resta là (avec donc des formes comme xan [San] 'manger', à côté de xori [Sori] 'oiseau'), en guipuscoan le passage de /S/ à /x/ n'affecta pas tous les /S/ : s'il toucha sans exception ceux provenant du glide /j/, d'autres se maintinrent, et on aboutit donc à jan [xan] d'un côté, et, de l'autre, après passage à l'affriquée de la chuintante initiale, à txori [tSori]. Comment rendre compte de ces données, si l'on résiste à admettre, pour des raisons bien compréhensibles, que les phonèmes conservent la mémoire de leur formation diachronique? Trask indique que la solution avancée par Michelena consiste à poser que la conservation du trait d'expressivité rattaché à l'emploi de la palatale en dehors des emprunts et de l'évolution mentionnée du glide d'avant, aurait bloqué la vélarisation de la fricative. Cette explication est considérée par Trask comme impliquant un exemple extraordinaire de blocage d'un changement phonologique pour des raisons fonctionnelles (p. 157). Ceci est néanmoins douteux, ou ne correspond qu'à une partie du problème. En effet, si les anciennes chuintantes initiales sont devenues affriquées, mais pas celles issues du glide d'avant, il faut souligner que les premières pouvaient être d'origines diverses (Michelena 1961, [1976 : p. 194]), et par conséquent qu'elles n'étaient pas toujours issues de formes expressives. Cette situation est illustrée par des exemples tels que txautu 'laver' ou txerfa-tu 'greffer', respectivement apparentés à lat. sanum et insertare probablement.

Les sections consacrées à l'aspiration, aux groupes de consonnes et à l'accentuation résument très bien les principaux éléments connus, avec les inconvénients inhérents aux synthèses sur des sujets parfois fort complexes (la question concernant l'accentuation, en particulier, étant traitée de manière quelque peu elliptique, en dépit des importants progrès accomplis dans ce domaine au cours de ces dernières années ; cf. Hualde (1997). Les lecteurs apprécieront le résumé des principaux changements phonologiques intervenus au cours des siècles (p. 166-169) et présentés dans un tableau des phonèmes du pré-basque selon les principales positions (initiale, finale et intervocalique). Deux sections novatrices doivent être mentionnées, l'une concernant la phonotactique (p. 172-176), l'autre la structure morphématique (p. 176-178), ainsi qu'une discussion très suggestive concernant la question du grand nombre de vocables à initiale vocalique (p. 179-183).

Le chapitre 4 est consacré à la grammaire, c'est-à-dire principalement ici à l'analyse diachronique de divers morphèmes grammaticaux. Quelques observations sur des points de détail : la forme ancienne des pronoms intensifs du type neu ou zeu discutées (p. 197) a dû avoir un - $r$ final, comme cela est attesté encore chez Axular (neurk et zeurk à l'ergatif), et ainsi que le montrent les formes intensives comme neuror ou nerau. A propos de la formation de l'article -a, dont l'origine dans le démonstratif distal est rappelée, Trask propose d'en dater $l^{\prime}$ introduction entre le $8^{\mathrm{e}}$ et le $10^{\mathrm{e}}$ siècle (p. 199), compte tenu, d'une part, de son absence dans les inscriptions aquitaines mais de sa présence dans le témoignage d'A. Picaud ( $12^{\mathrm{e}}$ siècle), et, d'autre part, de la datation de son apparition dans les langues romanes et germaniques. Concernant le suffixe -ero, principalement employé au sud dans des expressions indiquant un repère temporel (astero 'chaque semaine'), Trask propose de le rapprocher du suffixe adverbial -ro (p. 211). Ceci est peu probable car la distribution de -ro (qui se joint à des adjectifs ou des adverbes) est différente de celle de -ero (qui se joint uniquement à des noms marquant un repérage temporel); ce dernier suffixe paraît plutôt apparenté au quantifieur universel oro 'tout, tous' utilisé comme tel dans les dialectes orientaux.

Le point principalement discuté dans les indications concernant les morphèmes casuels est celui de la déclinaison locative, à propos duquel Trask propose le scénario suivant (p. 204) : le système actuel utilisé avec les noms propres refléterait le système le plus ancien (- $n$ marque d'inessif, -a marque d'adlatif). 
Plus tard, un morphème locatif apparenté avec le nom gain 'sommet' aurait été employé, qui lors de l'introduction de l'article et des oppositions de nombre aurait été interprété comme singulier. Ce morphème apparaît aujourd'hui dans le - $a$ - des formes ines-sives (qui dériverait de *gan comme proposé par Jacobsen (1977) et de Rijk (1981)). Pour les formes non singulières, le suffixe collectif -eta (emprunté probablement au latin) aurait été employé, et décliné à l'inessif, d'où -etan. Ce collectif aurait été interprété comme un pluriel, et ensuite le e- initial lui même aurait pris cette valeur (par analogie avec le - $e$-des cas obliques grammaticaux), de telle sorte que -ta- serai* devenu disponible pour l'indéfini. Comme le reconnaît Trask lui-même, l'hypothèse est largement spéculative, et on demeure circonspect, en particulier en ce qui concerne l'analyse de -ta, qui est employé dans tous les dialectes à l'indéfini, alors que le -e- pluriel n'est employé que dans les dialectes orientaux dans les cas obliques grammaticaux. Les données rapportées concernant les formes de l'adlatif en souletin sont passablement confuses. Trask (p. 206) relève la présence d'une étrange collection de désinences : -la, -lat, -alat, -ialat, -ilat. En fait le souletin a la même désinence que les dialectes voisins : $-r a(t)$, réalisée $-a(t)$ ou, après -a-, - $l a(t)$, cette dernière possibilité étant également attestée plus à l'ouest, en bas-navarrais, dans des formes comme eskuaralat (peut être par dissimilation) ou elizalat (Lafitte 1944 : § 140). Ce qui est propre au souletin, c'est la possibilité d'utiliser le morphème alocatif-a- de l'inessif à l'adlatif singulier également. On a donc etxera (qui correspond à la forme inessive etxen) et *etxiara(t) (qui correspond à l'inessif etxian) 'à la maison'. Après -a- l'adlatif étant toujours réalisé -la on a *etxiara $(t)>$ etxiala $(t)$; cf. Gèze (1 873 : p. 23) : Etxera(t) joan da et Etxiala joan da 'Il est allé à la maison'. Dans cette dernière forme -a- peut tomber (phénomène également rencontré en dehors des formes locatives avec les formes singulières de l'ergatif ou même le pluriel absolutif), et il en résulte alors etxila(t), tout comme l'on a mendian > mendin ou lanian > lanin. En fait, donc, l'alternance - $r a$ / -la est simplement conditionnée par la présence au singulier d'un a précédant le suffixe adlatif dans la dérivation, -la étant toujours exclu en dehors de ce contexte; par exemple dans les noms propres sans article ou avec les verbes nominalisés des infinitives: * Lakarrila(t) 'à Lacarry', *ikustela 'à voir'. Ceci donne à penser que /-est secondaire ici, contrairement à ce que suggère Trask, et contrairement aussi peut-être au suffixe -la à valeur modale rencontré avec les interrogatifs (nola 'comment', bisc. zelan), les pronoms démonstratifs (hala 'ainsi'), et le suffixe de flexion de certaines phrases subordonnées (dugula '(alors) que nous avons'). Concernant ce dernier suffixe, Trask (p. 211), curieusement, ne suggère aucun rapprochement avec l'adlatif (cf. Lafitte $1944: \S 757$ ).

En présentant les formes non finies du verbe, l'auteur reprend (p. 213) des analyses antérieures (Trask 1990) sur les verbes à radical en $-n$. L'idée consiste à supposer que les formes actuelles proviennent d'anciennes formes en - $n-i$ (avec -i aspectuel) : eman > *mani 'donner'. La chute du -n- intervocalique du participe passé aurait conduit à une confusion des formes radicale (eman) et participe (emai). Par la suite eman aurait permis de rendre tant le radical que le participe passé, et emai serait demeuré dans les seules formes radicales du type emai- rencontrées dans les dérivés nominaux (emaile, emaite). L'absence du - $n$ final du radical dans les formes conjugués s'expliquerait par sa chute dans les formes relatives (*dagonen) et du passé (* zegonen) en position intervocalique; explication qui rendrait compte de la syllabicité du complémenteur et des marques du passé après les radicaux dans les formes conjuguées (par opposition à ce qu'il advient lorsque ces suffixes se joignent aux marqueurs de personnes). Très ingénieuse, l'analyse laisse malgré tout certains points non expliqués, comme l'absence de $-/$ dans les dérivés nominaux de certains verbes : jan \#* jaite, edan \# *edaite, etzan \# *etzaite, ent-zun \#*entzuite, etc ; et surtout elle repose sur une analyse impliquant la chute du $-n$ - intervocalique dans un cadre particulier: celui de la morphologie flexionnelle. 
Par ailleurs l'explication, implicitement, accorde aux formes participes perfectives une grande importance (puisque que c'est à partir d'elles que la base lexicale des verbes est modifiée). Pourtant cette importance devait être moins grande dans la vieille langue que dans celle d'aujourd'hui, où les formes radicales ne sont plus disponibles que dans des formes marquées du point de vue modal (changement intervenu assez tardivement, au $16^{\mathrm{e}}$ siècle). Il est vrai que Trask (p. 238) pense que les formes aoristiques du type erran zezan 'il dit' sar zedin 'il entra', très nombreuses chez Liçarrague, représentent plutôt des innovations idiosyncrasiques, très localisées, que des survivances de la vieille langue, comme on le pense généralement (Lafon 1944). En réalité ces formes sont attestées dans tous les textes du $16^{\mathrm{e}}$ siècle: Dechepare, Liçarrague, les Refranes y Sentencias et également dans les proverbes d'Oihenart ; tout indique par conséquent que cet usage, contrairement à ce qu'indique Trask, était général jusqu'au $16^{\mathrm{e}}$ siècle ; (cf. pour les données en vieux bis-cayen, Lakarra 1986).

L'examen des formes verbales finies est également très clair. Trask fait le point sur la plupart des questions essentielles (analyse des préfixes personnels ou temporels, limites de l'accord tripersonnel, etc.). Parmi les points discutables, mentionnons: la surprenante indication (p. 221) selon laquelle, dans les dialectes du nord et de l'est, l'accord en nombre ne se ferait pas avec les objets indéfinis sémantiquement pluriels : Bi liburu dut /*ditut (sic) 'j'ai deux livres'. S'il est exact que cet accord n'est pas systématique en souletin, il est requis dans les plupart des dialectes du nord avec les quantifieurs cardinaux, et nulle part alors il n'est agrammatical. Cet accord pluriel est observable dès le $16^{\mathrm{e}}$ siècle, chez Dechepare et Liçarrague. On s'étonnera également de l'emploi de la désignation de pluralisateur ergatif, et de pluralisateur transitif à propos du suffixe -e (pas uniquement biscayen, loin s'en faut) ou te, se joignant à l'indice suffixé de troisième personnes ergative. Ce même suffixe s'emploie en réalité avec les datifs (e $<o / a+e$; -ote) et même comme pluralisateur de la $2^{\mathrm{e}}$ personne (quel que soit le cas du NP correspondant). Par ailleurs, il n'est pas exact que egon 'se trouver, rester' soit le seul verbe intransitif où ce pluralisateur apparaît comme il est indiqué (p. 222), puisqu'il apparaît avec les verbes izan 'être' (les formes comme girade 'nous sommes' ou dirade 'ils sont' sont déjà présentes dans les textes du $16^{\mathrm{e}}$ siècle, et plus à l'ouest garade apparaît dans le Salve transcrit par Isasti ; cf. Michelena 1964: p. 104) et sans doute également, *edin [n-/h-/d-adi- vs $g-/ z-/ d-a(d) i$ te-). Les indications concernant les préfixe affirmatif $b a$ - sont confuses (p. 226). En effet, pour illustrer la tendance des dialectes du nord à utiliser $b a$ - avec les formes synthétiques (même sans être en tête de phrase), Trask indique que pour dire 'Il est à la maison' les locuteurs de ces dialectes utiliseront aussi bien Etxean da que Etxean bada. Ceci n'est pas exact, et l'exemple est vraiment mal choisi, car s'il est un verbe où il n'est pas indifférent d'utiliser $b a$-, c'est précisément le verbe izan 'être', lequel, avec $b a$-, est ordinairement prédicat d'existence, et sans $b a$-, par contre, verbe copule. Le contraste est donc très marqué, dans les dialectes du nord, entre Ura da 'C'est de l'eau', et Ura bada 'Il y a de l'eau'. Une autre indication qu'il convient de rectifier concerne le génitif des objets directs des phrases nominalisées. Trask (p. 244) indique que cet emploi n'est pas attesté au sud des Pyrénées, alors qu'au contraire il l'est sans aucun doute possible dans la vieille langue biscayenne (Orpustan 1992, Lakarra 1997). 
Après l'analyse de ces divers morphèmes, où manque malgré tout une présentation des morphèmes de subordination, essentiellement liés en basque du point de vue diachronique, soit aux marques casuelles pour - $(e) n$ (cf. avec des points de vue divergents : Gavel 1929, de Rijk 1972), soit au morphème de prise en charge énonciative bai 'oui' pour ba- et bait- (Lafon 1966), l'auteur, dans la dernière section de chapitre (p. 245-247), propose un tableau synthétique de l'évolution typologique de la langue à partir de l'hypothèse selon laquelle les caractères typologiques du basque connu (langue SOV, système casuel, postpositions) seraient secondaires par rapport au système inverse (cf. dans le même sens Gómez \& Sainz 1995). L'ergativité aurait accompagné le passage d'un système VO au système OV, l'évolution postérieure ayant conduit à l'apparition d'une syntaxe accusative, sans que la morphologie ergative ne soit perdue. La perspective générale est intéressante, et même séduisante, bien qu'elle soit évidemment hors de portée de toute réfutation empirique, et donc, comme le dit Trask lui-même, forcément spéculative.

Le chapitre 5 consacré au lexique est le plus long (109 p.) et c'est celui qui intéressera sans doute le plus les comparatistes, car bien structuré autour d'une quinzaine de sections qui permettent de faire le point sur les éléments lexicaux les plus significatifs dans une perspective principalement historique (mais incluant certains éléments modernes). Le chapitre se termine par une présentation des 200 mots de la liste de Swadesh avec leur traduction basque.

Les composés et les dérivés sont présentés de manière très succincte, mais claire, malgré ici encore quelques détails à revoir : -keta ne se joint pas aux adjectifs (p. 253) et l'exemple donné par Trask (berriketa 'bavardage') est construit sur le nom berri 'nouvelle', et non sur l'adjectif signifiant 'neuf, récent'. Les traits généraux de phonétique expressive, les voies majeures des emprunts lexicaux viennent ensuite. La tradition néologique basque est l'objet d'une analyse essentiellement consacrée aux efforts aranistes et postérieurs. L'évocation de Larramendi au début de cette section est quelque peu allusive et surtout contestable car il est estimé que les composés créés par Larramendi étaient usuellement construits d'une manière techniquement correcte (p. 263). Au contraire, tel n'était pas le cas dans le dictionnaire trilingue (1749), et c'est avec Larramendi que se créé en fait dans la tradition lexicographique basque une confusion entre, d'une part, l'exploitation de ce qu'on appelle aujourd'hui parfois le lexique potentiel, déjà bien commencée dans leur travaux lexicaux par Harriet (1741) (cf. Lakarra 1995), ou Urte (cf. Urkizu 1987), et, d'autre part, la néologie savante (qui ne s'en tient pas aux seules règles régissant la formation du lexique basque, lesquelles sont d'ailleurs totalement ignorées jusqu'au $20^{\mathrm{e}}$ siècle). De ce point de vue Arana se situe dans la tradition larramendienne, même s'il s'en éloigne en ce que Larramendi n'a pas une attitude révisionniste, c'est-à-dire visant à corriger les vocables basques déjà formés, au nom du purisme lexical. Concernant le mot euzkadi créé par Arana, Trask indique qu'il fut formé sur euzko lui même créé à partir de eguzki 'soleil' (p. 264) : [eguz-kiko > euzko] + di > euzkadi. Cette explication est celle qui est généralement restituée en se fondant sur les indications données par Arana lui-même. Mais on sait désormais grâce à Zabaltza (1997) que l'histoire est sensiblement différente : le mot euzkadi en réalité est antérieur à euzko, et ce dernier vocable, lorsqu'il est créé par Arana, trouve sa source dans l'étymologie de bizkaia < be-euzko-di-a, où $b e$ - est 'bas'et où le composé s'entend 'l'ensemble des Basques du bas'. C'est à cause de cette étymologie à partir de bizkaia que fut changée l'orthographe de eusko, euskera, etc. avec substitution de -z- à -s-. Et ce n'est que cinq ans plus tard qu'Arana avança l'étymologie euzko $<$ eguzkiko. 
Les comparatistes tireront grand profit des sections consacrées au lexique spécialisé, qui est examiné de manière systématique par Trask au travers d'une cinquantaine de pages (p. 267-320) : sont ainsi successivement étudiés les noms de couleurs et de parenté, les numéraux, le lexique des jours, des mois, des saisons, des parties du corps, des instruments de base, d'orientation, celui des verbes de possession et d'être, de mouvement, les noms d'animaux, de végétaux, de concepts ou phénomènes atmosphériques ou géographiques, de nourriture, d'occupation et activités. Une partie du vocabulaire ethno-culturel est également abordé : noms de la langue, touchant à la mythologie, au folklore, à la vie politique ou culturelle. L'auteur fait le point de manière tout à fait satisfaisante et en général solidement documentée de la longue tradition concernant la formation de ce vocabulaire : jours de semaines, couleurs, partie du corps. En dehors de points de détails sur l'analyse de tel ou tel vocable (au total certainement plusieurs centaines), si une critique devait être apportée, elle porterait sur le fait que la distinction n'est pas établie entre ce qui relève du lexique effectivement employé, et des mots savants ou tardivement fabriqués par les lexicographes. Le point n'est pas crucial car Trask en ce cas se réfère en fait soit à des formations motivées, morphologiquement transparentes comme arraultzetegi pour 'ovaire', soit à des composés analogiques, descriptifs ou calqués, tels que gorputzenbor 'tronc ; litt. corps-tronc'(enbor étant utilisé en réalité pour les arbres), eguzkisorketa 'lever du soleil' (dont l'authenticité ou la 'naturalité' est passablement douteuse, contrairement à eguzki sortze), ou ehun-zango 'millepatte'(cf. esp. ciempiés, quoique ce dernier calque soit effectivement employé dans certains parlers, et attesté en dehors des dictionnaires).

Les hydronymes (p. 329-331), oronymes (p. 331-332), toponymes (p. 332-339), patronymes (p. 339-348) et domonymes (p. 349-352) sont ensuite présentés à travers un nombre relativement important d'exemples qui permettent au lecteur de se faire une idée précise sur la façon dont le basque aborde ces champs spécifiques, même si l'information est nécessairement condensée. L'auteur regrette (p. 349) que personne n'ait entrepris une étude systématique des noms de maison, ignorant visiblement certains travaux réalisés dans ce domaine, en particulier la thèse d'Orpustan (1984) demeurée, il est vrai, non publiée jusqu'ici.

Le dernier chapitre est consacré à la question des relations (principalement) génétiques avec d'autres familles linguistiques. On sait combien le sujet a été débattu, et combien aussi ces travaux ont donné lieu, et continuent de donner lieu, à des propositions, formulées généralement en termes définitifs par des personnes sans formation ou connaissances sérieuses en linguistique historique basque (et même, parfois, aucune en linguistique et guère plus en basque, ou inversement). Ce qui est un certainement excellent moyen de se prémunir des détestables préjugés scientistes, et de faire preuve d'audace et de hardiesse dans les hypothèses, mais ce qui aboutit inévitablement, en cette matière comme dans les autres branches du savoir, au ridicule ou à l'escroquerie éditoriale (l'un n'empêchant pas l'autre). Telle n'est pas la situation de Trask, qui a publié dictionnaires et ouvrages généraux de linguistique historique (cf. supra), et qui a acquis au cours des années une solide compétence en basque (ses premières publications relatives au basque remontent à plus de 20 ans), en particulier dans les domaines qui sont spécialement en jeu dans les travaux comparatistes (typologie, études diachroniques). 
Contrairement à beaucoup de linguistes, il n'est pas a priori hostile ou indifférent aux travaux de comparaison sur la longue distance du type de ceux développés par certains nostraticiens, et, malgré de très sérieux désaccords de méthode, il n'ignore pas les travaux se prévalant des méthodes de la comparaison multilatérale (Ruhlen 1987), comme l'a montré le débat publié dans la revue Mother Tongue (1995 et 1996) à partir de la critique de Trask des travaux réalisés dans le cadre de l'hypothèse dite macro-caucasique (pour une brève présentation dans une publication facile d'accès en Pays Basque, cf. Bengtson 1996), et plus généralement de la proposition visant à faire appartenir le basque à un macrophylum dit dénécau-casique. Enfin n'étant pas basque lui-même, on ne peut donc le soupçonner d'abriter quelque tendance obscure, induite par une fierté ou une susceptibilité nationale déplacée, à refuser à prendre en considération les éléments faisant perdre au basque son splendide (?) isolement dans le panorama linguistique européen. Libre donc d'un tel tropisme, que nous dit Trask sur cette question?

Dès le début de ce chapitre il met en garde le lecteur des tentations de ce qu'il appelle la comparaison bongo-bongo, consistant à rapprocher en vertu de leurs ressemblances, réelles ou supposées, des mots basques avec d'autres, pris dans telle ou telle autre langue, selon les convenances (p. 358). L'accent est donné et le chapitre est une charge implacable des diverses tentatives effectuées jusqu'ici en vue d'établir sans trop de précautions des liens génétiques entre le basque et telle ou telle langue ou famille linguistique connue, en se fondant sur de simples rapprochements lexicaux. La leçon est rude, et en dehors de la section consacrée à l'aquitain (p. 398-403), où ses vues se situent dans la lignée de celles de Michelena (1961/1976) et Gorrochategui (1984), l'exercice tient beaucoup du jeu de massacre. La grande majorité des linguistes sera certainement sensible aux arguments de l'auteur qui se situe dans le cadre théorique de la linguistique historique classique, et qui par conséquent insiste sur les exigences minimales que l'on est en droit d'attendre des auteurs dans l'établissement de liens génétiques entre une langue $\mathrm{X}$ et une langue ou une famille de langues $\mathrm{Y}$ demeurées en tout état de cause sans aucun contact durant plusieurs milliers d'années. Je me limiterai ici à indiquer quelles hypothèses Trask présente dans cet examen : tentatives opérées dans le cadre de la glottochronologie (p. 359-361), parenté avec des langages d'Afrique et notamment les langues berbères et la famille afro-asiatique (p. 361 -364), parenté avec les autres langues de l'Europe pré-indoeuropéenne (p. 364-368), parenté avec la famille indoeuropéenne (p. 368378), liens avec la langue ibérique (p. 378-388), le minoen ancien (p. 388-390), la langue des Pictes (p. 390-392), les langues du Caucase (p. 392-398), l'hypothèse déné-caucasique et 'proto-mondiale' (p. 403-411). Comme on le voit, le parcours est assez complet. En est simplement absente l'hypothèse finno-ougrienne et sa variante macro, dite ouralo-altaïque, qui dispose également d'une tradition ancienne dans les études basques et qui a été reprise récemment (Morvan 1992). Il ne fait guère de doute que le jugement de Trask concernant cette hypothèse ne serait guère différent de celui émis à propos des autres rapprochements mentionnés ci-dessus. Au demeurant, pour faire la démonstration par l'absurde des dangers d'une méthodologie reposant sur la seule ressemblance lexicale, il offre une liste de 65 vocables (p. 412-414) paraissant démontrer une claire affinité entre le basque et le hongrois.

Le chapitre se termine par quelques considérations de linguistique comparative aréale, Trask se livrant à un examen critique de la thèse, fort répandue dans la tradition romaniste ibérique, d'une influence du basque dans la formation du castillan. 
Le livre offre également, comme il se doit, un index général incluant les toponymes et les patronymes, les noms de langues, et quelques éléments thématiques. Mais on aurait apprécié qu'y fût joint également, de manière séparée, un index des vocables basques cités. L'ouvrage, devenu d'ores et déjà une pièce indispensable de la bibliothèque des études comparatives et diachroniques basques, le méritait.

\section{B. Oyharçabal \\ CNRS, UPRESA 5478}

\section{BIBLIOGRAPGHIE}

Abbadie, A. \& Chaho, J. A. 1836. Études grammaticales sur la langue euskarienne, Paris.

Aizpuma, X. 1995. La continuité de la langue basque, Eusko Jaurlaritza, Vitoria.

Arotçarena, Abbé -. 1951. Grammaire basque (Dialectes Navarro-Labourdins), Le Porche, Bayonne.

Artiagoitia, X. A par. « Determinatzaile Sintagmaren Hipotesia Euskal Gramatikan ».

Bengtson, B. 1996. «Correspondances of Basque and Caucasic Final Stem Vowels . -il/-e, $-u /-o », F L V, 71,07-15$.

Coyos, J.-B. 1998. Description du parler basque souletin des Arbailles. Étude de l'ergativité, thèse de doctorat, Université Paris V, non publiée.

Darrigol, J.-C. 1827. Dissertation critique et apologétique sur la langue basque, Bayonne.

OEH-DGV. Orotariko Euskal Hiztegia-Diccionario General Vasco, 10 tomes depuis 1987, Euskaltzaindia, Bilbao.

Gavel, H. 1920. Éléments de phonétique basque, Champion, Paris.

Gavel, H. 1929. Grammaire basque, tome 1, Bayonne.

Gómez, R. \& Sainz, K. 1995. « On the Origin of the Finite Forms of the Basque Verb », in J. I. Hualde, J. Lakarra \& R. L. Trask (édit.) Towards a History of the Basque Language, John Benjamins, Amsterdam/Philadelphie, 235-274.

Gorrochategui, J. 1984. Estudio sobre la onomástica indigena de Aquitania, Euskal Herriko Unibertsitatearen argitarapen zerbitzua.

Gorrochategui, J. 1995. «Basque and its neighbors in Antiquity », in J. I. Hualde, J. Lakarra \& R. L. Trask (édit.) Towards a History of the Basque Language, John Benjamins, Amsterdam/Philadelphie, 31-64.

Harriet, M. 1741. Gramatica escuaraz eta francesez composatua frances hitzcuntça ikhasi nahi dutenen faboretan, Fauvet, Bayonne. 
Hualde, J. I. Euskararen azentuerak, Anejos del Anuario del Seminario de Filologia Vasca 'Julio de Urquijo', XLII, Saint-Sébastien.

Hurch, B. 1991. "Sobre la reconstrucción del euskera. Observaciones a Trask », in J. Lakarra \& I. Ruiz (édit.) Memoriae L. Michelena Magistri Sacrum, 2 vol., Anejos del Anuario del Seminario de Filologia Vasca 'Julio de Urquijo', XIV, Saint-Sébastien, Pars altera : 607614.

Jacobsen, W. H. 1977. « The Basque locative Suffix », in W. Douglass, R. Etulain \& W. Jacobsen (édit.), Anglo-American Contributions to Basque Studies : Essays in Honor of Jon Bilbao, Reno.

Lafitte, P. 1944. Grammaire basque (navarro-labourdin littéraire), édition revue et corrigée, Amis du Musée Basque et Ikas, Bayonne, 1962.

Lafon, R. 1933. «Sur le traitement de $u$ devant le groupe 'r plus consonne'en souletin », RIEV, XXIV.

Lafon, R. 1944. Le système du verbe basque au XVIe siècle, édition fac-similé Elkar, Bayonne, 1980.

Lafon, R. 1966. "La particule BAIT en basque; ses emplois morphologiques et syntaxiques », BSL, LXI, 131-159.

Lakarra, J. 1986. «Bizkaiera zaharra gainerako euskalkien artean », Anuario del Seminario de Filologia Vasca 'Julio de Urquijo', XX-3, 639-681.

Lakarra, J. 1995. Harrieten gramatikako hiztegiak (1741), Anejos del Anuario del Seminario de Filologia Vasca 'Julio de Urquijo ', XVIII, Saint-Sébastien.

Lakarra, J. 1997. Refranes y Sentencias (1596). Ikerketak eta edizioa, Euskaltzaindia, Bilbao.

Lécluse, F. 1826. Manuel de la langue basque, édition par J. Lakarra dans Anuario del Seminario de Filologia Vasca 'Julio de Urquijo', 1987, XXI-3, Saint-Sébastien.

Levin, B. On the Nature of ergativity, Thèse, MIT.

Martinet, A. 1955. Économie des changements phonétiques, Berne.

Martinet, A. 1958. «La construction ergative et les structures élémentaires de l'énoncé », Journal de Psychologie, 377-392.

Michelena, L. 1961. «Euskal hiztegigileak XVII-XVIIIgarren mendeetan », Euskera, VI, 7-22.

Michelena, L. 1961/1976. Fonética histórica vasca, deuxième édition corrigée et augmentée, Diputacion de Guipúzcoa, Saint-Sébastien. 
Michelena, L. 1964. Textos arcáicos vascos, Minotauro, Madrid ; édition facsimilé Anejos del Anuario del Seminario de Filologia Vasca 'Julio de Urquijo', XI, 1990, Saint-Sébastien.

Morvan, M. 1992. Les origines linguistiques du basque: l'ouralo-altä̈que, Thèse de doctorat, Université de Bordeaux 3. Version remaniée publiée sous le titre Les origines linguistiques du basque, 1996, Presses universitaires de Bordeaux.

Nichols, J. 1992. Linguistics diversity in Space and Time, University of Chicago Press, Chicago.

N'Diaye, G. 1970. Structure du dialecte de Maya, Mouton, La Haye - Paris.

Orpustan, J.-B. 1992. «Un trait dialectal en basque moderne et contemporain: le complément au génitif du nom verbal », in Iker-7, Actes du congrès de dialectologie tenu à Bilbao du 21 au 25 octobre 1991, Euskaltzaindia, Bilbao, 535-558.

Orpustan, J.-B. 1984. Les noms de maisons médiévales en Soule, Labourd et BasseNavarre. Étude de toponymie et histoires médiévales, thèse de doctorat, Université de Bordeaux 3, non publiée ; (deux ouvrages rassemblant la substance de ce travail sont à paraître dans les prochains mois).

Ortiz de Urbina, J. \& Uribe-Etxebarria, M. 1991. «Participial predication in Basque », in J. Lakarra \& I. Ruiz (edit.) Memoriae L. Michelena Magistri Sacrum, 2 vol., Anejos del Anuario del Seminario de Filologia Vasca 'Julio de Urquijo', XIV, Saint-Sébastien, Pars Altera : 9931012.

Oyharçabal, B. 1997. «La situation de la langue basque en Pays Basque nord», Lapurdum, 2, 29-44.

Rebuschi, G. 1983/1984. Structure de l'énoncé en basque, thèse de doctorat (Université Paris 7, 1982), SELAF, Paris.

Rijk de —, R. 1981. «Euskal morfologiaren zenbait gorabehera », in Euskal linguistika eta literatura: bide berriak, (sans nom d'éditeur), Deustuko Unibertsitatea - Universidad de Deusto, Bilbao.

Ruhlen, M. 1987. A Guide to the World's Languages, vol. I: Classification, Edward Arnold, Londres, Melbourne, Auckland.

Tchekhoff, C. 1978. Aux fondements de la syntaxe : l'ergatif Presses Universitaires de France, Paris.

Trask, R. L. 1985a. " On the reconstruction of the pre-Basque phonology », in J. Melena (édit.) Symbolae Ludovico Michelena Septuagenario Oblatae, 2 vol. Instituto de Ciencias de la Antigüedad, Universidad del PaísVasco, 885-881.

Trask, R. L. 1985b. «-ko atzizkia euskaraz », Euskera, 1985, 30, 165-174.

Trask, R. L. 1990. «The $-n$ class of verbs in Basque », Transactions of the Philological Society, 88, 11-128. 
Trask, R. L. 1993. A Dictionary of Grammaticals Terms in Linguistics, Routledge, Londres/New York.

Trask, R. L. 1994. Linguistic Change, Routledge, Londres/New York.

Urkizu, P. 1987. Piarres d'Urteren hiztegia, thèse de doctorat, Euskal Herriko Unibertsitatea - Universidad del Pais Vasco, Vitoria-Gasteiz.

Zabaltza, X. 1997. «El significado oculto de la palabra Euzkadi », FLV, n 74, 77-84.

NoTE

Les données sont assez complexes, et varient non seulement selon les dialectes, mais même à l'intérieur des dialectes selon les vocables. La tendance générale est à la perte de l'opposition soit du fait de l'amuïssement du $r$ simple (le souletin offrant le cas extrême puisque le phénomène concerne également la position intervocalique), soit du fait d'une neutralisation au profit d'une réalisation correspondant à $r r$. Toutefois le système ne s'est pas partout stabilisé, et il n'est pas impossible que la réalisation uvulaire de $r r$ dans les parlers du nord ait aidé à maintenir l'opposition dans certains vocables à $r$ final doux. Ainsi pour de nombreux locuteurs actuel du Labourd et de Basse-Navarre, il y a une opposition nettement marquée entre zenbat ur [ur]'combien d'eau'et zenbat ur [uR]'combien de noisettes'. Pour des indications montrant l'existence de cette même opposition dans les parlers orientaux du sud, voir Michelena (1961/1976 : 334, n. 13). 